\title{
A VORTICITY MODEL FOR VISCOUS FLOW PAST A CYLINDER
}

\author{
S. J. D. D'Alessio and S. C. R. Dennisł \\ Department of Applied Mathematics, University of Western Ontario, London, \\ Ontario, Canada N6A 5B7
}

(Received 24 November 1992; in revised form 12 April 1993)

\begin{abstract}
A mathematical model is proposed for the steady two-dimensional flow of a viscous incompressible fluid past a cylinder which incorporates the details of the structure of the vorticity in this case where its behaviour is known. The model is constructed to be consistent both with boundary-layer theory for sufficiently large Reynolds numbers and with the asymptotic solution at large distances from the cylinder. The governing Navier-Stokes equations are transformed to a set of equations which we refer to as the modified Navier-Stokes equations and are then solved numerically. Solutions have been obtained for the cases of flow past a stationary and a rotating circular cylinder and fiow past an inclined elliptic cylinder. Good agreement is found with existing results.
\end{abstract}

\section{INTRODUCTION}

The steady two-dimensional flow of a viscous incompressible fluid past a cylindrical body represents a classical problem in fluid mechanics. Exact analytical solutions are still out of reach owing to the non-linearities in the governing Navier-Stokes equations. Although the numerical solution to the symmetrical problem has been addressed by several authors, relatively few have attempted the asymmetrical case. As pointed out by Dennis [1], one of the main difficulties encountered lies in the satisfactory treatment of boundary conditions at large distances from the cylinder. In the present work the behaviour of the vorticity is patterned for both small and large distances by modelling it to comply with boundary-layer and wake theory, respectively. This is done by employing a transformation which ensures the correct decay of vorticity and satisfaction of boundary conditions at large distances from the cylinder, but is also consistent with boundary-layer theory close to it.

Most of the previous investigations of flow past a cylinder have employed coordinate systems which transform the solution domain into a semi-infinite open rectangular strip with the cylinder located at the closed end of the rectangle. Examples are the polar coordinate system for a circular cylinder and elliptic coordinates for flow past an elliptic cylinder. In such coordinate systems the wake region in which the vorticity is significant is concentrated within a region whose width becomes smaller as the distance from the cylinder increases. This creates difficulties when computational methods using finite differences are employed to obtain solutions, since very small grids must be used to capture the details of the solution in the far wake. Badr et al. [2], in fact, used a series of successive grid reductions in the wake in computing steady flow past a rotating cylinder, but without attempting to stretch the breadth of the wake. In the present work we deal with this problem by applying a transformation that removes the part of the solution which causes the wake to narrow. In effect this is modelled on the classical theory of the wake as described, for example, by Imai [3]. However, the variables employed in the wake are not generally suitable near the cylinder, so the transformation incorporates an element which rectifies this situation.

In order to apply the transformation it is necessary to employ a coordinate system which is appropriate to the particular cylinder cross-section under investigation. This is briefly described in the following section. Then, in subsequent sections, the modified form of the vorticity transport equation is obtained and the basic ideas behind the model are identified. A numerical method of solving the model equation is described and applied to the problem of determining the steady flow

†Author for correspondence. 
past a non-rotating circular cylinder, a rotating circular cylinder and an elliptic cylinder inclined to the oncoming stream.

\section{BASIC EQUATIONS AND BOUNDARY CONDITIONS}

Expressed in terms of the stream function $\psi$ and the vorticity $\zeta$, the governing Navier-Stokes equations in Cartesian coordinates in dimensionless form are given by

$$
\frac{\partial^{2} \psi}{\partial x^{2}}+\frac{\partial^{2} \psi}{\partial y^{2}}+\zeta=0
$$

and

$$
\frac{\partial^{2} \zeta}{\partial x^{2}}+\frac{\partial^{2} \zeta}{\partial y^{2}}=\frac{\operatorname{Re}}{2}\left(\frac{\partial \psi}{\partial y} \frac{\partial \zeta}{\partial x}-\frac{\partial \psi}{\partial x} \frac{\partial \zeta}{\partial y}\right)
$$

where the dimensionless parameter $\mathrm{Re}$ is the Reynolds number and is related to the oncoming fluid velocity $U$, the typical length $d$ and the kinematic viscosity $v$ according to $\operatorname{Re}=2 U d / v$.

At this point it is convenient to introduce the conformal transformation

$$
x+i y=H(\xi+i \theta),
$$

which transforms the contour of the cylinder to $\xi=\xi_{0}$ and the infinite region exterior to the cylinder to the semi-infinite rectangular strip $\xi \geqslant \xi_{0},-\pi \leqslant \theta \leqslant \pi$. The mapping (3) is assumed to be of Joukowski type, chosen in such a way that it has the asymptotic form given by the equations

$$
x \sim \mathrm{ke}^{\xi} \cos \theta, \quad y \sim \mathrm{ke}^{\xi} \sin \theta,
$$

where the constant $\mathrm{k}$ is dependent on the cylinder cross-section.

In terms of the transformed coordinates $(\xi, \theta)$ the equations of motion (1) and (2) become

$$
\frac{\partial^{2} \psi}{\partial \xi^{2}}+\frac{\partial^{2} \psi}{\partial \theta^{2}}+M^{2} \zeta=0
$$

and

$$
\frac{\partial^{2} \zeta}{\partial \xi^{2}}+\frac{\partial^{2} \zeta}{\partial \theta^{2}}=\frac{\operatorname{Re}}{2}\left(\frac{\partial \psi}{\partial \theta} \frac{\partial \zeta}{\partial \xi}-\frac{\partial \psi}{\partial \xi} \frac{\partial \zeta}{\partial \theta}\right)
$$

where $M$ refers to the metric of the transformation and can be found through

$$
M^{2}=\left(\frac{\partial x}{\partial \xi}\right)^{2}+\left(\frac{\partial x}{\partial \theta}\right)^{2}
$$

Boundary conditions for $\psi$ and $\zeta$ for the problems considered include the no-slip condition

$$
\psi=0, \frac{\partial \psi}{\partial \xi}=-\Omega \quad \text { when } \quad \xi=\xi_{0},
$$

where in the case of a rotating circular the dimensionless angular velocity $\Omega=d \omega_{0} / U$, with $\omega_{0}$ denoting the dimensional angular velocity in the counter-clockwise direction. For a stationary cylinder $\Omega=0$; in this case, conditions (8) are appropriate for a cylinder of arbitrary cross-section placed in a uniform stream. By periodicity we have that

$$
\psi(\xi, \theta)=\psi(\xi, \theta+2 \pi), \quad \zeta(\xi, \theta)=\zeta(\xi, \theta+2 \pi)
$$

while the free-stream conditions demand that

$$
\frac{\partial \psi}{\partial \xi} \sim \mathrm{ke}^{\xi} \sin \theta, \quad \frac{\partial \psi}{\partial \theta} \sim \mathrm{ke}^{\xi} \cos \theta, \quad \zeta \rightarrow 0 \text { as } \xi \rightarrow \infty .
$$

By inspecting the boundary conditions we see that there are two conditions for the stream function on the cylinder surface, while none for the vorticity. Different methods for handling this situation have been developed and will be discussed later. Further, imposing conditions (10) at a 
large but finite distance away is too crude. Instead, the asymptotic solutions for $\psi$ and $\zeta$ are used at $\xi=\xi_{\infty}$, some large enough value of $\xi$. In the event of symmetrical flow both $\psi$ and $\zeta$ are odd functions of $\theta$ and the solutions of equations (5) and (6) are required only in the region $0 \leqslant \theta \leqslant \pi$ subject to the conditions

$$
\psi=\zeta=0 \quad \text { when } \theta=0, \pi
$$

\section{A VORTICITY MODEL}

It is known that the vorticity is only significant in a thin boundary layer adjacent to the cylinder surface and within a parabolic wake. Outside of these regions the flow is essentially inviscid. Based on this information a model for the vorticity can be formulated. By expressing the vorticity as

$$
\zeta(\xi, \theta)=\Phi(\xi, \theta) \mathrm{e}^{F \xi, \theta)}
$$

and by judiciously choosing the function $F(\xi, \theta)$, the vorticity can be forced to comply with known asymptotics and boundary-layer theory. Further, the function $\Phi$ can be designed to be roughly the same order of magnitude throughout the entire flow field. This a good feature to have when doing numerical work.

From the asymptotic solution of Imai [3] we demand that

$$
F(\xi, \theta) \sim-\frac{\operatorname{Re} \mathrm{k}}{2} \mathrm{e}^{\xi}(1-\cos \theta) \quad \text { for large } \xi .
$$

We shall see that this ensures a slow decay of $\Phi$ as $\xi \rightarrow \infty$, which is more suitable for computation than the very rapid decay of the vorticity $\zeta$. On the other hand, boundary-layer theory suggests that

$$
\xi=0\left(\frac{1}{\sqrt{\mathrm{Re}}}\right) \quad \text { for small } \xi \text { and sufficiently large Re. }
$$

A function appropriate to both of these constraints is given by

$$
F(\xi, \theta)=\frac{\mathrm{Rek}}{2}(\cosh \xi-1)(\cos \theta-1) .
$$

Thus, outside the boundary-layer and far wake the vorticity will be exponentially small.

Many physical properties of the flow can be extracted from this model. For example, the decay of the vorticity is dictated by the exponential factor $\mathrm{e}^{F(\xi, \theta)}$. By plotting

$$
\exp \left[\frac{\operatorname{Rek}}{2}(\cosh \xi-1)(\cos \theta-1)\right]=\epsilon
$$

for some small specified $\epsilon$, curves indicating the extent to which the vorticity is confined can be generated for various Re. Such curves appear to be similar to those of Fornberg [4] which were obtained by a mapping and reveal the following information:

(a) the vorticity is confined to a smaller region as Re increases;

(b) vorticity is convected far downstream, while upstream it is diffused very short distances;

(c) the fluid can be considered to be inviscid beyond the curves; and

(d) asymptotic boundary conditions for the vorticity can be applied close to the cylinder upstream but must be applied far from the cylinder within the wake.

In addition, we shoose to work in terms of the perturbed stream function $\psi^{*}$, defined through

$$
\psi=\psi^{*}+2 \mathbf{k} \sinh \xi \sin \theta
$$


again to ensure that $\psi^{*}=0(1)$ throughout the flow field. In other words, we employ a function $\psi^{*}$ that is well-behaved as $\xi \rightarrow \infty$. Expressions (12) and (17), when substituted into equations (5) and (6), yield for $\psi^{*}$ and $\Phi$ the two equations:

$$
\frac{\partial^{2} \psi^{*}}{\partial \xi^{2}}+\frac{\partial^{2} \psi^{*}}{\partial \theta^{2}}+M^{2} \mathrm{e}^{F} \Phi=0
$$

and

$$
\begin{aligned}
& \frac{\partial^{2} \Phi}{\partial \xi^{2}}+\frac{\partial^{2} \Phi}{\partial \theta^{2}}-\left(\operatorname{Rek} \sinh \xi+\frac{\operatorname{Re}}{2} \frac{\partial \psi^{*}}{\partial \theta}\right) \frac{\partial \Phi}{\partial \xi}+\left(\operatorname{Rek} \sin \theta+\frac{\operatorname{Re}}{2} \frac{\partial \psi^{*}}{\partial \xi}\right) \frac{\partial \Phi}{\partial \theta} \\
& +\frac{\operatorname{Rek}}{2}\left[(\cos \theta-\cosh \xi)+\frac{\operatorname{Re}}{2} \sinh \xi(1-\cos \theta) \frac{\partial \psi^{*}}{\partial \theta}-\frac{\operatorname{Re}}{2}(\cosh \xi-1) \sin \theta \frac{\partial \psi^{*}}{\partial \xi}\right] \Phi=0 .
\end{aligned}
$$

We refer to equations (18) and (19) as the modified Navier-Stokes equations. The boundary conditions ( 8 ) and (9) now become

$$
\psi^{*}=-2 \mathrm{k} \sinh \xi_{0} \sin \theta, \quad \frac{\partial \psi^{*}}{\partial \xi}=-\Omega-2 \mathrm{k} \cosh \xi_{0} \sin \theta \quad \text { when } \xi=\xi_{0}
$$

and

$$
\psi^{*}(\xi, \theta)=\psi^{*}(\xi, \theta+2 \pi), \quad \Phi(\xi, \theta)=\Phi(\xi, \theta+2 \pi)
$$

The asymptotic conditions as $\xi \rightarrow \infty$ are dealt with in the following section.

\section{ASYMPTOTICS OF THE MODIFIED NAVIER-STOKES EQUATIONS}

At large distances the modified Navier-Stokes equations can be linearized by making use of the Oseen approximations:

$$
\frac{\partial \psi^{*}}{\partial \xi} \sim 0, \quad \frac{\partial \psi^{*}}{\partial \theta} \sim 0 \quad \text { for large } \xi
$$

This leads to the modified Oseen equations:

$$
\frac{\partial^{2} \psi^{*}}{\partial \xi^{2}}+\frac{\partial^{2} \psi^{*}}{\partial \theta^{2}}+M^{2} \mathrm{e}^{F} \Phi=0
$$

and

$$
\frac{\partial^{2} \Phi}{\partial \xi^{2}}+\frac{\partial^{2} \Phi}{\partial \theta^{2}}-\operatorname{Rek} \sinh \xi \frac{\partial \Phi}{\partial \xi}+\operatorname{Rek} \sin \theta \frac{\partial \Phi}{\partial \theta}-\frac{\operatorname{Rek}}{2}(\cosh \xi-\cos \theta) \Phi=0 .
$$

These linear decoupled equations are valid for large $\xi$ for any Re. However, for small Re they are uniformly valid throughout the entire flow field.

Equation (24) can be solved analytically by first performing the transformation

$$
\Phi(\xi, \theta)=\exp \left[\frac{\operatorname{Rek}}{2}(\cosh \xi+\cos \theta)\right] \chi(\xi, \theta) .
$$

This removes all first-derivative terms and yields an equation for $\chi$, given by

$$
\frac{\partial^{2} \chi}{\partial \xi^{2}}+\frac{\partial^{2} \chi}{\partial \theta^{2}}-\frac{\operatorname{Re}^{2} \mathbf{k}^{2}}{4}\left(\cosh ^{2} \xi-\cos ^{2} \theta\right) \chi=0 .
$$

By separation of variables the general solution of equation (26) involves the periodic even-odd Mathieu functions $c e_{n}(\theta)$ and $s e_{n}(\theta)$, respectively, and the corresponding modified Mathieu 
functions $\mathrm{Fe}_{n}(\xi)$ and $\mathrm{Gek}_{n}(\xi)$. Thus,

$$
\begin{aligned}
\Phi(\xi, \theta)=\exp \left[\frac{\operatorname{Rek}}{2}(\cosh \xi\right. & +\cos \theta)] \\
& \times\left\{a_{0} F e k_{0}(\xi) c e_{0}(\theta)+\sum_{n=1}^{\infty}\left[a_{n} F e k_{n}(\xi) c e_{n}(\theta)+b_{n} \operatorname{Gek}_{n}(\xi) s e_{n}(\theta)\right]\right\} .
\end{aligned}
$$

Using the asymptotic expressions for $F_{e} k_{n}(\xi)$ and $\operatorname{Gek}_{n}(\xi)$, we arrive at

$$
\Phi(\xi, \theta) \sim P(\theta) \mathrm{e}^{-\xi / 2} \text { as } \xi \rightarrow \infty,
$$

where $P(\theta)$ denotes some function of $\theta$. The asymptotic expression for $\psi^{*}$ will be the same as that given by Imai [3], namely

$$
\psi^{*}(\xi, \theta) \sim \frac{C_{\mathrm{D}}}{2}\left[\frac{\theta}{\pi}-\operatorname{erf}\left(\sqrt{\frac{\operatorname{Rek}}{2}} \mathrm{e}^{\xi / 2} \sin \frac{\theta}{2}\right)\right]+\frac{C_{\mathrm{L}}}{2 \pi} \ln \left(\mathrm{ke}^{\xi}\right)+\text { e.s.t. } \quad \text { as } \xi \rightarrow \infty .
$$

Here, $C_{\mathrm{D}}$ and $C_{\mathrm{L}}$ designate the drag and lift coefficients, respectively, while $\operatorname{erf}(\phi)$ refers to the error function [5, p. 295] and e.s.t. denotes an exponentially small term. It may be noted that approximation (28) follows directly from equation (19) subject to approximations (22), since the leading terms as $\xi \rightarrow \infty$ give the balance

$$
\frac{1}{2} \operatorname{Rek~e}\left(\frac{\partial \Phi}{\partial \xi}+\frac{1}{2} \Phi\right) \sim 0
$$

\section{METHOD OF SOLUTION}

The modified Navier-Stokes equations (18) and (19) are solved by finite differences using a Gauss-Seidel iterative procedure with under-relaxation applied only to the surface vorticity. The modified vorticity transport equation (19) can be rewritten in the form

$$
\frac{\partial^{2} \Phi}{\partial \xi^{2}}+\frac{\partial^{2} \Phi}{\partial \theta^{2}}+2 \lambda \frac{\partial \Phi}{\partial \xi}+2 \mu \frac{\partial \Phi}{\partial \theta}-\gamma \Phi=0
$$

where

$$
\begin{aligned}
& \lambda(\xi, \theta)=-\frac{\operatorname{Re}}{4}\left(2 \mathrm{k} \sinh \xi+\frac{\partial \psi^{*}}{\partial \theta}\right), \\
& \mu(\xi, \theta)=\frac{\operatorname{Re}}{4}\left(2 \mathrm{k} \sin \theta+\frac{\partial \psi^{*}}{\partial \xi}\right)
\end{aligned}
$$

and

$$
\gamma(\xi, \theta)=\frac{\operatorname{Rek}}{2}\left[(\cosh \xi-\cos \theta)-\frac{\operatorname{Re}}{2} \sinh \xi(1-\cos \theta) \frac{\partial \psi^{*}}{\partial \theta}+\frac{\operatorname{Re}}{2}(\cosh \xi-1) \sin \theta \frac{\partial \psi^{*}}{\partial \xi}\right] .
$$

The computational domain for symmetrical flow, bounded by $\xi_{0}<\xi<\xi_{\infty}$ and $0<\theta<\pi$, is discretized into a network of $(N-1) \times(M-1)$ grid points located at

$$
\xi_{i}=\xi_{0}+i h, \quad i=1,2, \ldots, N-1,
$$

and

$$
\theta_{j}=j k, \quad j=1,2, \ldots, M-1,
$$

with

$$
h=\frac{\xi_{\infty}-\xi_{0}}{N}
$$


and

$$
k=\frac{\pi}{M}
$$

For asymmetrical flow the computational domain bounded by $\xi_{0}<\xi<\xi_{\infty}$ and $-\pi<\theta<\pi$ is discretized by a similar network of $(N-1) \times(2 M-1)$ grid points. In this case

$$
\theta_{j}=j k, j=-(M-1),-M, \ldots,(M-1) .
$$

Because $\lambda \sim-(\operatorname{Re} / 4) \mathrm{e}^{\xi}$ for large $\xi$ it is clear that diagonal dominance, a sufficient condition for the convergence of a Gauss-Seidel procedure, will certainly be violated in that region of the flow field. For this reason the upwind-differencing scheme was adopted to solve equation (31). In this scheme the terms $\partial \Phi / \partial \xi$ and $\partial \Phi / \partial \theta$ are approximated by forward or backward differences, depending upon the signs of $\lambda$ and $\mu$, while central differencing is used on the remaining terms. The discretization of equation (31) then leads to

$$
c_{0} \Phi_{i, j}=c_{1} \Phi_{i+1, j}+c_{2} \Phi_{i, j+1}+c_{3} \Phi_{i-1, j}+c_{4} \Phi_{i, j-1},
$$

where the notation $\Phi_{i, j}=\Phi\left(\xi_{i}, \theta_{j}\right)$ is used. Here,

$$
\begin{array}{lll}
c_{1}=1+2 h \lambda_{i, j}, & c_{3}=1 & \text { if } \lambda_{i, j}>0, \\
c_{1}=1, & c_{3}=1-2 h \lambda_{i, j} & \text { if } \lambda_{i, j}<0, \\
c_{2}=h^{2}\left(1+2 \mathrm{k} \mu_{i, j}\right) / \mathrm{k}^{2}, & c_{4}=h^{2} / \mathrm{k}^{2} & \text { if } \mu_{i, j}>0, \\
c_{2}=h^{2} / \mathbf{k}^{2}, & c_{4}=h^{2}\left(1-2 \mathrm{k} \mu_{i, j}\right) / \mathrm{k}^{2} & \text { if } \mu_{i, j}<0
\end{array}
$$

and

$$
c_{0}=2\left[1+h\left|\lambda_{i, j}\right|+h^{2}\left(1+\mathbf{k}\left|\mu_{i, j}\right|\right) / \mathbf{k}^{2}+h^{2} \gamma_{i, j} / 2\right] .
$$

Although this technique is only first-order accurate, a deferred correction term can be added to the right-hand side of equation (40) to render the procedure second-order accurate. This term is given by $D_{i, j}=d_{i}+d_{j}$, where

$$
d_{i}=-\frac{h\left|\mu_{i, j}\right|}{2}\left(\Phi_{i+1, j}+\Phi_{i-1, j}-2 \Phi_{i, j}\right), \quad d_{j}=-\frac{h^{2}\left|\lambda_{i, j}\right|}{2 \mathrm{k}}\left(\Phi_{i, j+1}+\Phi_{i, j-1}-2 \Phi_{i, j}\right) .
$$

Replacing all derivatives in equation (18) by central differences results in the following second-order finite-difference scheme:

$$
2\left(1+\frac{h^{2}}{\mathrm{k}^{2}}\right) \psi_{i, j}^{*}=\psi_{i+1, j}^{*}+\psi_{i-1, j}^{*}+\frac{h^{2}}{\mathrm{k}^{2}}\left(\psi_{i, j+1}^{*}+\psi_{i, j-1}^{*}\right)+h^{2} M_{i, j}^{2} \exp \left(F_{i, j}\right) \Phi_{i, j} .
$$

An iteration of the procedure involves sweeping through all grid points using equations (40) and (43). After each iteration $C_{\mathrm{D}}$ and $C_{\mathrm{L}}$ are updated. These coefficients are defined by equations (46) and (47) for a circular cylinder and by equations (55) and (56) for an elliptic cylinder in the following section. Convergence is reached when successive values fall within some specified tolerance.

As mentioned earlier, careful consideration must be given to the boundary conditions used at large distances from the cylinder. Using asymptotic conditions along the line $\xi=\xi_{\infty}$ is equivalent to the assumption that beyond this line the flow is governed by the modified Oseen equations. Provided $\xi_{\infty}$ is taken large enough, this assumption will be valid. For the vorticity we implement the gradient condition

$$
\Phi\left(\xi_{\infty}, \theta\right)=\Phi\left(\xi_{\infty}-h, \theta\right) \mathrm{e}^{-h / 2},
$$

which is based on approximation (28), while for the perturbed stream function expression (29) is used on $\xi=\xi_{\infty}$. 


\section{CALCULATED RESULTS}

Numerical solutions have been obtained for the cases of a stationary and rotating circular cylinder and an inclined elliptic cylinder. For the special case of symmetric flow, solutions were also obtained using the method of Dennis and Chang [6] and the agreement was excellent. In their method the surface vorticity is found by utilizing integral or global conditions generated by the application of Green's second identity.

\subsection{Flow past a circular cylinder}

For the circular cylinder $\xi_{0}=0, \mathrm{k}=1, H(\xi+i \theta)=\exp (\xi+i \theta)$ and $M^{2}=\mathrm{e}^{2 \xi}$. The flow can be completely characterized by the parameters $\operatorname{Re}$ and $\Omega$. The surface vorticity was obtained by a second-order finite-difference expression derived by taking a Taylor expansion of $\psi^{*}$ about the cylinder surface and making use of the extra condition for $\psi^{*}$. This local method yields the equation

$$
\Phi_{0, j}=\left\{-\frac{1}{3}\left(4 \Phi_{1, j}-\Phi_{2, j}\right)-\frac{4 \psi_{1, j}^{*}}{h^{2}}-\frac{4}{h}\left[\Omega+2\left(1+\frac{h^{2}}{6}\right) \sin \theta\right]\right\} /\left(1+\frac{4 h}{3}\right) .
$$

The above expression can also be used for the symmetrical case by setting $\Omega=0$.

The symmetric problem was solved first. This was then used as an initial guess for the asymmetric problem for a small rotation rate. The rotation rate was then stepped using the previous value as the initial guess. This was done only to accelerate the convergence of the numerical procedure. Although the Gauss-Seidel procedure was used to solve the difference equations throughout the field in general, it was found to be necessary to use under-relaxation to calculate the surface vorticity with a relaxation parameter as low as 0.05 . This is quite usual, although care must be taken to ensure that true convergence has been obtained.

The drag and lift coefficients were computed numerically on the cylinder surface using the formulae

$$
C_{\mathrm{D}}=\frac{2}{\operatorname{Re}} \int_{-\pi}^{\pi}\left(\frac{\partial \zeta}{\partial \xi}-\zeta\right)_{0} \sin \theta \mathrm{d} \theta
$$

and

$$
C_{\mathrm{L}}=\frac{2}{\operatorname{Re}} \int_{-\pi}^{\pi}\left(\zeta-\frac{\partial \zeta}{\partial \xi}\right)_{0} \cos \theta \mathrm{d} \theta
$$

The solutions were tested by checking the constancy of $C_{\mathrm{D}}$ and $C_{\mathrm{L}}$ for integration paths off the surface and the periodicity of the pressure. These were found to be constant within reasonable limits, particularly near the cylinder surface.

Table 1 compares flow properties, such as the drag coefficient $C_{\mathrm{D}}$, the wake length $l_{\mathrm{w}}$ measured from the rear of the cylinder to the end of the separated region, the angle of separation $\theta_{s}$, where the surface vorticity vanishes, and the front and rear dimensionless stagnation point pressures $P_{0}(\pi)$ and $P_{0}(0)$, respectively, obtained by the present method with those of Dennis and Chang [6] and

Table 1. Comparison of flow properties for $\operatorname{Re}=5,20,40,100$ for flow past a circular cylinder with $\Omega=0$

\begin{tabular}{rllllll}
\hline Re & \multicolumn{1}{c}{ Reference } & $C_{\mathrm{D}}$ & $P_{0}(0)$ & $P_{0}(\pi)$ & $l_{\mathrm{w}}$ & $\theta_{\mathrm{s}}(\mathrm{deg})$ \\
\hline \multirow{2}{*}{5} & Present work & 3.809 & -0.940 & 1.838 & - & - \\
& Dennis and Chang [6] & 4.116 & -1.044 & 1.872 & - & - \\
20 & Present work & 1.941 & -0.536 & 1.264 & 1.75 & 43.1 \\
& Dennis and Chang [6] & 2.045 & -0.589 & 1.269 & 1.88 & 43.7 \\
& Fornberg [7] & 2.000 & -0.54 & 1.28 & 1.82 & - \\
\multirow{2}{*}{40} & Present work & 1.443 & -0.455 & 1.141 & 4.65 & 53.0 \\
& Dennis and Chang [6] & 1.522 & -0.509 & 1.144 & 4.69 & 53.8 \\
& Fornberg [7] & 1.498 & -0.46 & 1.14 & 4.48 & - \\
& Present work & 1.077 & -0.319 & 1.063 & 9.50 & 65.8 \\
& Dennis and Chang [6] & 1.056 & -0.393 & 1.060 & 13.11 & 66.2 \\
& Fornberg [7] & 1.058 & -0.34 & 1.06 & 12.7 & - \\
\hline
\end{tabular}




Table 2. Parameters used in the calculations for flow past a circular
cylinder with $\Omega=0$
\begin{tabular}{rccc}
\hline $\operatorname{Re}$ & Grid size $N \times M$ & $\xi_{x}$ & $\omega$ \\
\hline 5 & $80 \times 40$ & 4 & 0.05 \\
20 & $80 \times 40$ & 4 & 0.05 \\
40 & $120 \times 60$ & 4 & 0.025 \\
100 & $120 \times 90$ & 3.5 & 0.02 \\
\hline
\end{tabular}

Fornberg [7] for selected $\mathrm{Re}$ in the range $5 \leqslant \operatorname{Re} \leqslant 100$ for the case $\Omega=0$. These pressures were evaluated using the formulae

$$
P_{0}(\pi)=1+\frac{4}{\operatorname{Re}} \int_{0}^{\infty}\left(\frac{\partial \zeta}{\partial \theta}\right)_{\theta=\pi} \mathrm{d} \xi, \quad P_{0}(0)=1+\frac{4}{\operatorname{Re}} \int_{0}^{\infty}\left(\frac{\partial \zeta}{\partial \theta}\right)_{\theta=0} \mathrm{~d} \xi .
$$

In computing $P_{0}(0)$, the range of integration $(0, \infty)$ was broken up into the two intervals $\left(0, \zeta_{\infty}\right)$ and $\left(\xi_{\infty}, \infty\right)$. Because of the slow decay of the vorticity within the wake, the contribution resulting from the range $\left(\xi_{\infty}, \infty\right)$ is significant. To estimate this the asymptotic solution for $\zeta$ given by Imai [3] was used. This is consistent with the assumption that beyond the outer boundary $\xi_{\infty}$ the flow is adequately described by the asymptotic solution. Table 2 lists the parameters used in the calculations. The agreement found is reasonable, with the largest deviation occurring at $\operatorname{Re}=100$, as might be expected. However, Fornberg [7] used finer grids and the results of Dennis and Chang [6] were obtained using an $h^{4}$-accurate method. The critical Re at which separation first sets in was also determined. It was found to be in the range $6.39<\mathrm{Re}<6.40$ and agrees well with the value of 6.2 predicted by Dennis and Chang [6]. Computed streamlines for the case $\operatorname{Re}=100$ are shown in Fig. 1.

For the case of the rotating circular cylinder, numerical solutions were carried out for $\operatorname{Re}=5$, 20 with rotation rates in the range $0.1 \leqslant \Omega \leqslant 0.5$. These were the $R e$ values originally considered by Loc [8] and Ingham [9] and later by Badr et al. [2] and Ingham and Tang [10]. In the present work in all cases the grid size $N \times M=80 \times 60$, the relaxation parameter $\omega=0.05$ and $\xi_{x}=5$ (corresponding to approx. 150 radii) were found to be adequate. Several runs were carried out with

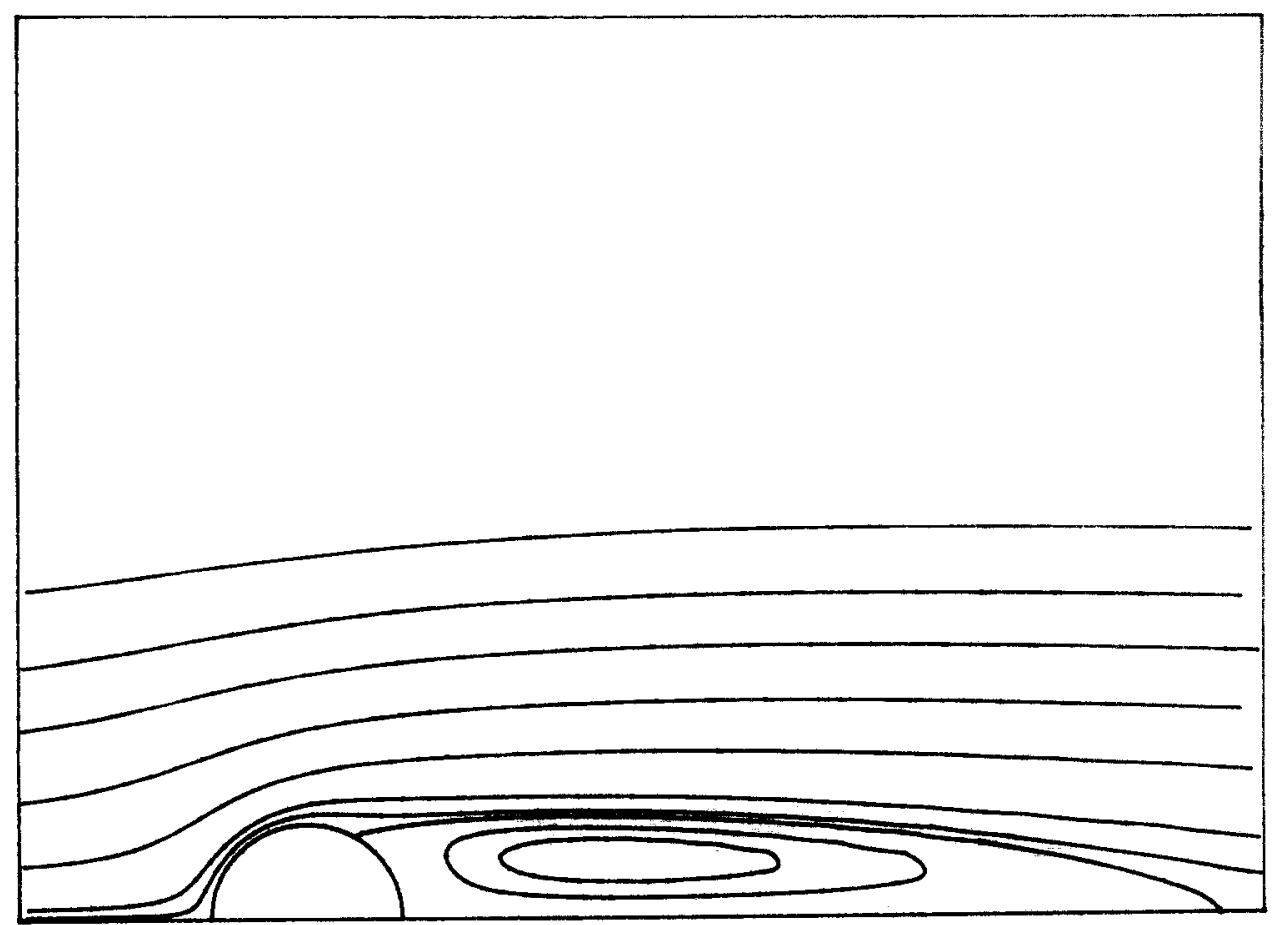

Fig. 1. Streamlines for $\operatorname{Re}=100$ and $\Omega=0$ for flow past a circular cylinder. 
Table 3. Comparison of $C_{\mathrm{L}}$ and $C_{\mathrm{D}}$ for a rotating circular cylinder

\begin{tabular}{|c|c|c|c|c|c|c|c|}
\hline \multirow[b]{2}{*}{$\operatorname{Re}$} & \multirow[b]{2}{*}{$\Omega$} & \multicolumn{2}{|c|}{ Badr et al. [2] } & \multicolumn{2}{|c|}{$\begin{array}{c}\text { Ingham and } \\
\text { Tang [10] }\end{array}$} & \multicolumn{2}{|c|}{ Present work } \\
\hline & & $-C_{\mathrm{L}}$ & $C_{\mathrm{D}}$ & $-C_{\mathbf{L}}$ & $C_{\mathrm{D}}$ & $-C_{\mathrm{L}}$ & $C_{\mathrm{D}}$ \\
\hline 5 & $\begin{array}{l}0.1 \\
0.2 \\
0.4 \\
0.5\end{array}$ & $\begin{array}{l}0.285 \\
0.572 \\
1.14 \\
1.37\end{array}$ & $\begin{array}{l}3.92 \\
3.91 \\
3.90 \\
3.89\end{array}$ & $\begin{array}{l}0.277 \\
0.559 \\
1.111 \\
1.389\end{array}$ & $\begin{array}{l}3.947 \\
3.939 \\
3.927 \\
3.916\end{array}$ & $\begin{array}{l}0.266 \\
0.533 \\
1.068 \\
1.336\end{array}$ & $\begin{array}{l}3.896 \\
3.893 \\
3.884 \\
3.877\end{array}$ \\
\hline 20 & $\begin{array}{l}0.1 \\
0.2 \\
0.4 \\
0.5\end{array}$ & $\begin{array}{l}0.266 \\
0.552 \\
1.11 \\
1.39\end{array}$ & $\begin{array}{l}2.00 \\
2.00 \\
1.97 \\
1.97\end{array}$ & $\begin{array}{l}0.254 \\
0.514 \\
1.024 \\
1.283\end{array}$ & $\begin{array}{l}1.995 \\
1.992 \\
1.979 \\
1.973\end{array}$ & $\begin{array}{l}0.243 \\
0.486 \\
0.974 \\
1.220\end{array}$ & $\begin{array}{l}1.916 \\
1.915 \\
1.912 \\
1.910\end{array}$ \\
\hline
\end{tabular}

different values of these parameters. For example, computations were performed with an $80 \times 40$ grid and also with the outer boundary positioned at $\xi_{\infty}=4.5$. It was found that the numerical results barely depended upon the grid size and only mildly depended upon the value of $\xi_{\infty}$. In particular, we quote the results:

(a) with $\operatorname{Re}=5, \Omega=0.1, N \times M=80 \times 40, \xi_{\infty}=4.5$ and $\omega=0.05, C_{\mathrm{D}}=3.918$ and $C_{\mathrm{L}}=-0.259$ were obtained;

(b) with $\operatorname{Re}=5, \Omega=0.1, N \times M=80 \times 60, \xi_{\infty}=4.5$ and $\omega=0.05, C_{\mathrm{D}}=3.912$ and $C_{\mathrm{L}}=-0.259$ were obtained; and

(c) with $\operatorname{Re}=5, \Omega=0.1, N \times M=80 \times 60, \xi_{\infty}=5$ and $\omega=0.05, C_{\mathrm{D}}=3.896$ and $C_{\mathrm{L}}=-0.266$ were obtained.

Convergence could have also been obtained for larger values of the relaxation parameter, expecially for the smaller Re values; however, this question was not studied here. In Table 3 comparisons of $C_{\mathrm{D}}$ and $C_{\mathrm{L}}$ are drawn with the results of Badr et al. [2] and Ingham and Tang [10]. Streamlines for this problem are portrayed in Figs 2 and 3. It is apparent from these diagrams that separation does not take place when the cylinder is allowed to rotate.

Badr et al. [2] use grid reduction in the far wake and scale the vorticity and stream function so that they are $0(1)$ in the far field. Asymptotic conditions are imposed at the outer boundary

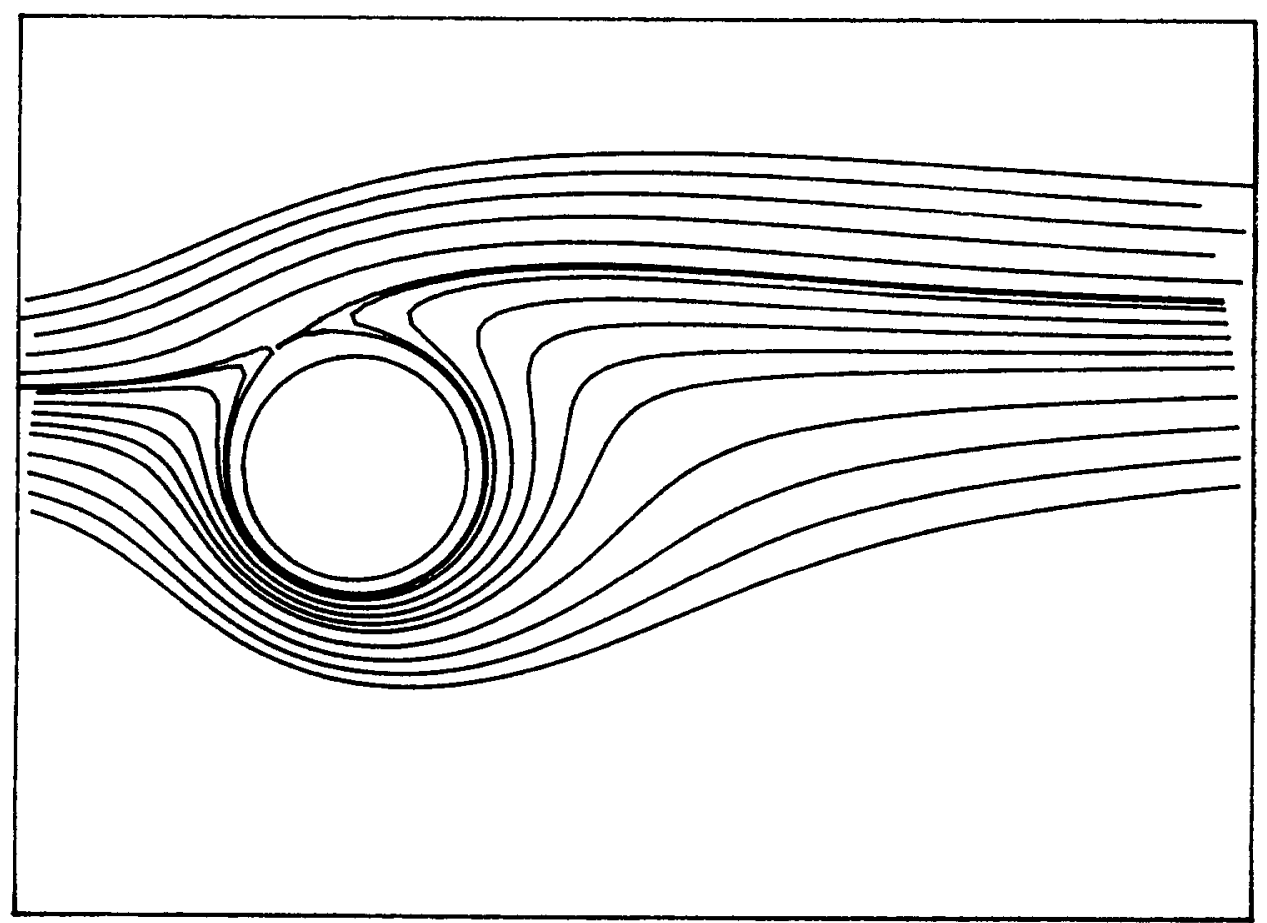

Fig. 2. Streamlines for $\operatorname{Re}=5$ and $\Omega=0.5$ for flow past a rotating circular cylinder. 


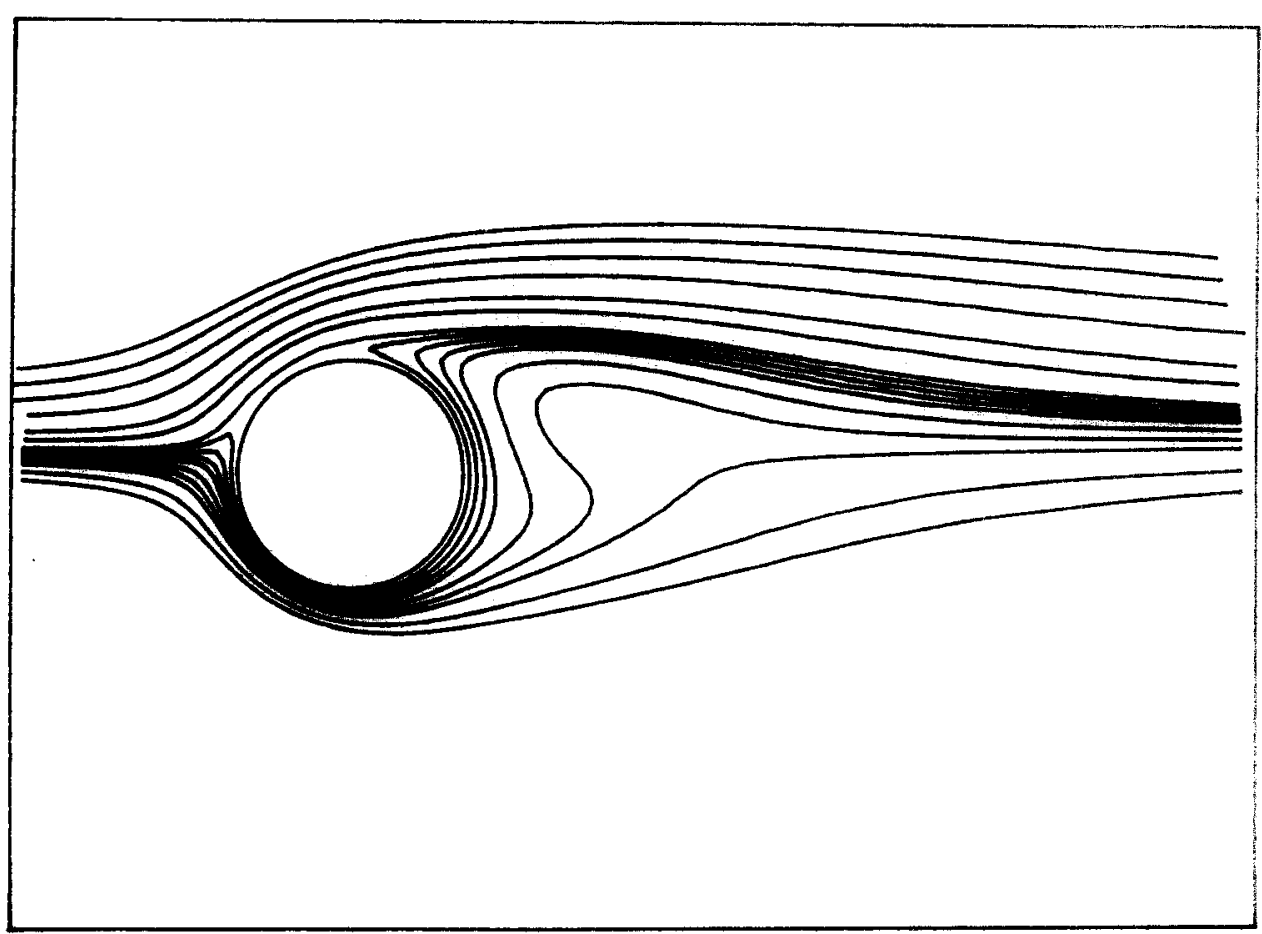

Fig. 3. Streamlines for $\mathrm{Re}=20$ and $\Omega=0.2$ for flow past a rotating circular cylinder.

approximating infinity. Ingham and Tang [10], on the other hand, propose a technique which avoids the need for asymptotic bounary conditions. This technique involves the transformation $\xi=1 / r$ which maps $r=\infty$ to $\xi=0$, and the function $f=\psi / r$ in place of the perturbed stream function $\psi$. The conditions then imposed on $\xi=0$ are $f=0$ and $\zeta=0$. Both of these authors also use a local $h^{2}$-accurate formula to compute the surface vorticity.

Finally, we may note that Tang and Ingham [11] have obtained steady-state solutions to this problem at $R e=60$ and 100 . On the other hand, Badr et al. [2] found that in an unsteady integration following an impulsive start from rest, vortex shedding from the cylinder started after some time had elapsed at both of these $\operatorname{Re}$ values, but shedding did not start at $\operatorname{Re}=5$ and 20 . It is clear that solutions of the steady-state equations can be obtained at higher values of Re, but we have not attempted this.

\subsection{Flow past an elliptic cylinder}

For the elliptic cylinder $k=1 / 2$,

$$
H(\xi+i \theta)=\cosh (\xi+i(\theta+\alpha)) \quad \text { and } \quad M^{2}=[\cosh 2 \xi-\cos (2(\theta+\alpha))] / 2 .
$$

Here, $\alpha$ denotes the inclination angle (or angle of attack) that the major axis makes with the uniform stream. The value of $\xi_{0}$ is determined from

$$
\tanh \xi_{0}=r,
$$

with $r$ specifying the ratio of the minor to major axis of the ellipse. Since the dimensionless length of the major axis is $2 \cosh \xi_{0}$ we define a Reynolds number, $\mathrm{Re}^{\prime}$, related to $\mathrm{Re}$ according to

$$
\operatorname{Re}=\frac{\operatorname{Re}^{\prime}}{\cosh \xi_{0}}
$$

Thus, for the elliptic cylinder, equation (49) is used for Re in equation (19) and everywhere else necessary. The flow is then characterized by the parameters $\mathrm{Re}^{\prime}, \alpha$ and $r$. 
A second-order finite-difference expression for the surface vorticity is given by

$$
\begin{aligned}
\{2 h[ & \left.\left.\sinh 2 \xi_{0}+\frac{\operatorname{Rek}}{2} M_{0, j}^{2} \sinh \xi_{0}(\cos \theta-1)\right]+3 M_{0, j}^{2}\right\} \Phi_{0, j} \\
= & M_{0, j}^{2}\left(\Phi_{2, j}-4 \Phi_{1, j}\right)+\frac{12}{h^{2}} \exp \left(-F_{0, j}\right)\left(\psi_{0, j}^{*}-\psi_{1, j}^{*}\right) \\
& \quad-\frac{12 \mathrm{k}}{h} \exp \left(-F_{0, j}\right) \sin \theta\left[\left(2+\frac{h^{2}}{3}\right) \cosh \xi_{0}+h \sinh \xi_{0}\right] .
\end{aligned}
$$

Again, under-relaxation of the surface vorticity was applied.

As before, computations were obtained by stepping the inclination angle, in a manner similar to that for the circular cylinder, using the symmetrical problem as the initial guess. After numerous trial runs, the following parameter values were found to be adequate: $\omega=0.05, \xi_{\infty}=5$ and $N \times M=100 \times 60$. Some test runs were carried out for a trial case, whereby the position of $\xi_{\infty}$ was placed further away from the cylinder. This parameter was varied because the results are more sensitive to changes in $\xi_{\infty}$ than any other parameter. In particular we quote the results:

(a) with $\operatorname{Re}^{\prime}=5, \alpha=10^{\circ}, r=0.2$ and $\xi_{\infty}=5, C_{\mathrm{D}}=2.751$ and $C_{\mathrm{L}}=0.452$ were obtained; and

(b) with $\operatorname{Re}^{\prime}=5, \alpha=10^{\circ}, r=0.2$ and $\xi_{\infty}=5.5, C_{\mathrm{D}}=2.755$ and $C_{\mathrm{L}}=0.466$ were obtained.

For the elliptic cylinder at inclination $\alpha$ to the free stream the dimensionless pressure and friction components are related to their dimensional counterparts by dividing by $\rho U^{2} d$ and are found to be:

$$
\begin{aligned}
& X_{\mathrm{P}}=\frac{\sinh 2 \xi_{0}}{\mathrm{Re}^{\prime}} \int_{-\pi}^{\pi}\left(\frac{\partial \zeta}{\partial \xi}\right)_{0} \sin (\theta+\alpha) \mathrm{d} \theta, \\
& X_{\mathrm{F}}=-\frac{2 \cosh ^{2} \xi_{0}}{\operatorname{Re}^{\prime}} \int_{-\pi}^{\pi} \zeta_{0} \sin (\theta+\alpha) \mathrm{d} \theta, \\
& Y_{\mathrm{P}}=-\frac{2 \cosh ^{2} \xi_{0}}{\operatorname{Re}^{\prime}} \int_{-\pi}^{\pi}\left(\frac{\partial \zeta}{\partial \xi}\right)_{0} \cos (\theta+\alpha) \mathrm{d} \theta
\end{aligned}
$$

and

$$
Y_{\mathrm{F}}=\frac{\sinh 2 \xi_{0}}{\mathrm{Re}^{\prime}} \int_{-\pi}^{\pi} \zeta_{0} \cos (\theta+\alpha) \mathrm{d} \theta .
$$

If we define $X=X_{\mathrm{P}}+X_{\mathrm{F}}$ and $Y=Y_{\mathrm{P}}+Y_{\mathrm{F}}$, we then arrive at

$$
C_{\mathrm{D}}=X \cos \alpha+Y \sin \alpha
$$

and

$$
C_{\mathrm{L}}=Y \cos \alpha-X \sin \alpha,
$$

for the expressions for the drag and lift coefficients.

The tests previously discussed for the circular cylinder were also performed on the numerical solutions of the elliptic cylinder and again the errors were tolerable. The results obtained for $r=0.2$ are in close agreement with the recent work of Dennis and Young [12] (which represents the only documented steady-state solutions for flow past an elliptic cylinder) and are displayed in Table 4. Dennis and Young [12] use the same numerical method as outlined in Ref. [2]. The essential object in their paper is to make a detailed study of how separation occurs with $\mathrm{Re}^{\prime}$ and inclination for the range $5 \leqslant \operatorname{Re}^{\prime} \leqslant 40$. There is exceptionally good agreement between their results at $\operatorname{Re}^{\prime}=5$, 20 and the present results. Streamlines are given in Figs 4 and 5 for the inclinations $\alpha=30^{\circ}$ and $70^{\circ}$ for the case $\operatorname{Re}^{\prime}=5$. No separation of the fluid takes place at $\alpha=30^{\circ}$ but as the inclination increases a separated region forms, as is just evident at $\alpha=70^{\circ}$. For the case $\operatorname{Re}^{\prime}=20$, shown in 


\begin{tabular}{|c|c|c|c|c|c|}
\hline \multirow[b]{2}{*}{$\mathrm{Re}^{\prime}$} & \multirow[b]{2}{*}{$\alpha$} & \multicolumn{2}{|c|}{$\begin{array}{l}\text { Dennis and } \\
\text { Young [12] }\end{array}$} & \multicolumn{2}{|c|}{ Present work } \\
\hline & & $C_{1}$ & $C_{\mathrm{D}}$ & $C_{1}$ & $C_{\mathrm{D}}$ \\
\hline \multirow[t]{10}{*}{5} & $0^{\prime \prime}$ & 0 & 2.712 & 0 & 2.713 \\
\hline & 10 & 0.451 & 2.754 & 0.452 & 2.751 \\
\hline & $20^{\prime}$ & 0.821 & 2.872 & 0.805 & 2.869 \\
\hline & 30 & 1.053 & 3.043 & 1.037 & 3.040 \\
\hline & 40 & 1.137 & 3.240 & 1.118 & 3.237 \\
\hline & 50 & 1.081 & 3.437 & 1.064 & 3.433 \\
\hline & $60^{\circ}$ & 0.908 & 3.610 & 0.904 & 3.614 \\
\hline & $70^{\circ}$ & 0.650 & 3.742 & 0.648 & 3.749 \\
\hline & $80^{\circ}$ & 0.338 & 3.825 & 0.337 & 3.834 \\
\hline & $90^{\circ}$ & 0 & 3.854 & 0 & 3.862 \\
\hline \multirow[t]{10}{*}{20} & 0 & 0 & 1.169 & 0 & 1.169 \\
\hline & $10^{\prime}$ & 0.420 & 1.202 & 0.426 & 1.204 \\
\hline & $20^{\circ}$ & 0.741 & 1.296 & 0.751 & 1.305 \\
\hline & $30^{\circ}$ & 0.915 & 1.436 & 0.923 & 1.452 \\
\hline & $40^{\circ}$ & 0.947 & 1.602 & 0.949 & 1.620 \\
\hline & $50^{\circ}$ & 0.865 & 1.766 & 0.866 & 1.785 \\
\hline & 60 & 0.706 & 1.911 & 0.706 & 1931 \\
\hline & $70^{\circ}$ & 0.498 & 2.022 & 0.497 & 2.045 \\
\hline & $80^{\circ}$ & 0.257 & 2.093 & 0.256 & 2.116 \\
\hline & $90^{\circ}$ & 0 & 2.119 & 0 & 2.140 \\
\hline
\end{tabular}

Figs 6 and 7, separation has started at the smaller inclination $\alpha=40^{\circ}$ and develops as $\alpha$ increases. In both cases of $\operatorname{Re}^{\prime}=5,20$ a symmetrical vortex pair is formed behind the cylinder for the inclination $\alpha=90^{\circ}$. There is extremely good comparisons between these figures and the corresponding ones of Dennis and Young [12].

Lastly, runs were performed whereby $r$ was allowed to approach zero so as the approximate a flat plate. Solutions were computed for the cases of flow at zero incidence and normal incidence for $\operatorname{Re}^{\prime}=20$. For the case of zero incidence with $r=0.02$, a drag of $C_{\mathrm{D}}=0.970$ was obtained which approaches the flat-plate solution $(r=0)$ of Dennis and Dunwoody [13], namely $C_{\mathrm{D}}=0.966$. For the problem of flow at normal incidence, good qualitative agreement is found with that of Hudson and Dennis [14] who solved an unsteady model problem of flow past a normal flat plate in order to reach steady state. Streamlines for $\alpha=90^{\circ}$ and $r=0.05$ are given in Fig. 8 .

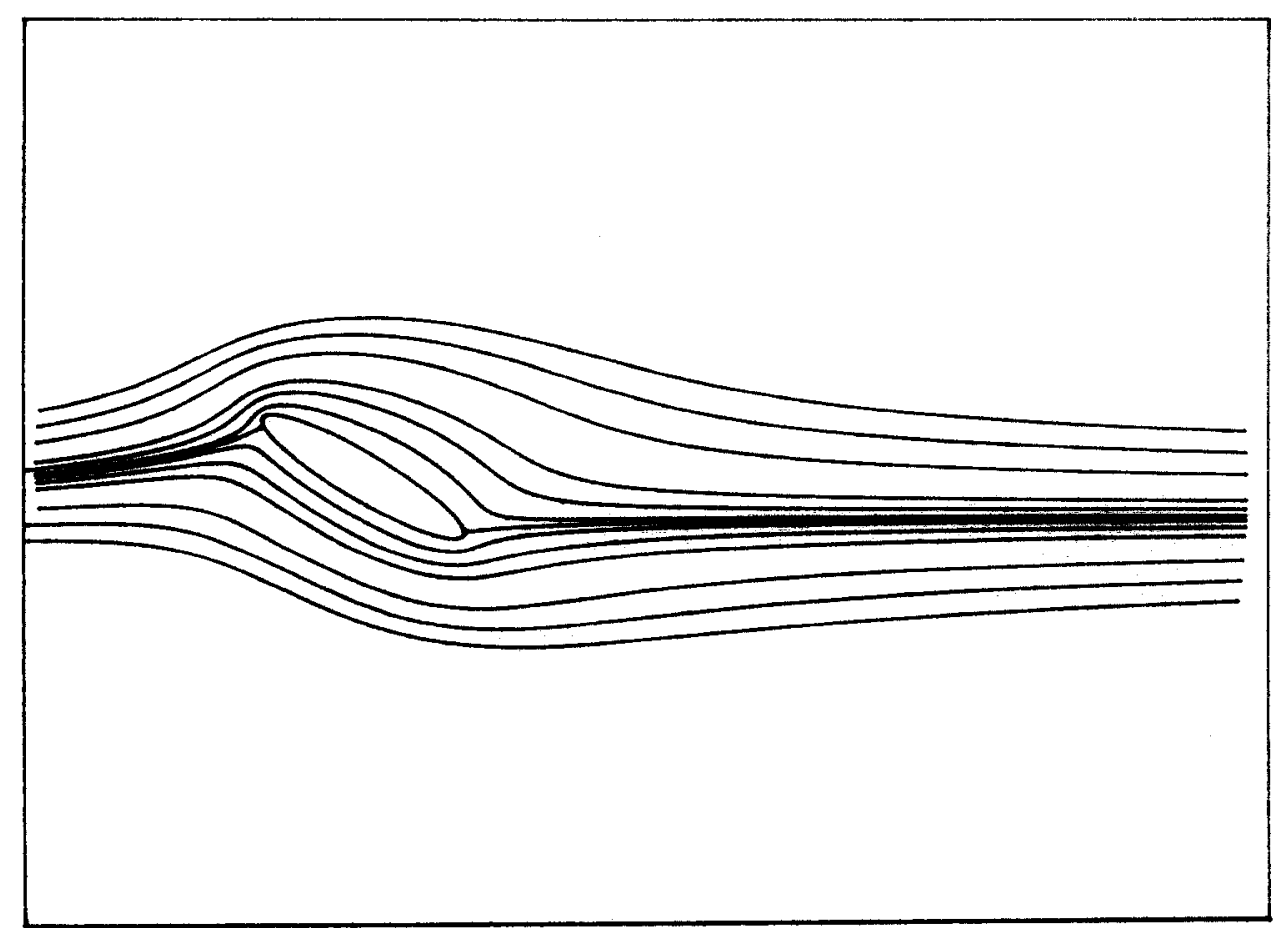

Fig. 4. Streamlines for $\operatorname{Re}^{\prime}=5, \alpha=30^{\circ}$ and $r=0.2$ for flow past an inclined elliptic cylinder. 


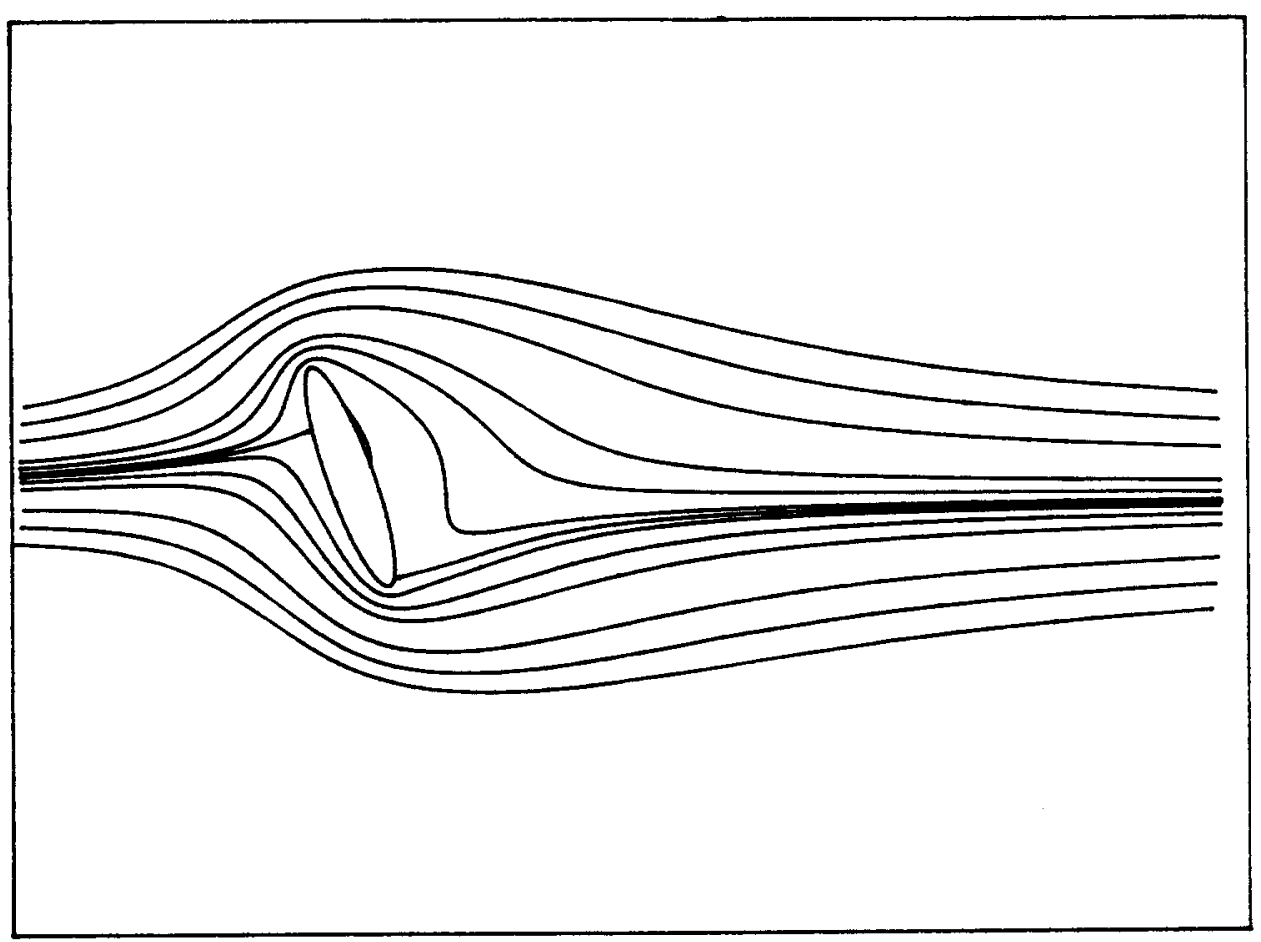

Fig. 5. Streamlines for $\operatorname{Re}^{\prime}=5, \alpha=70^{\circ}$ and $r=0.2$ for flow past an inclined elliptic cylinder.

\section{CONCLUSION}

In this paper we have described a model equation which may be used to compute the vorticity in the vorticity-stream function formulation of the two-dimensional Navier-Stokes equations for flow past a cylinder. The vorticity is transformed to a new dependent variable using a transformation which removes the rapid variation inherent in the untransformed variable at large distances

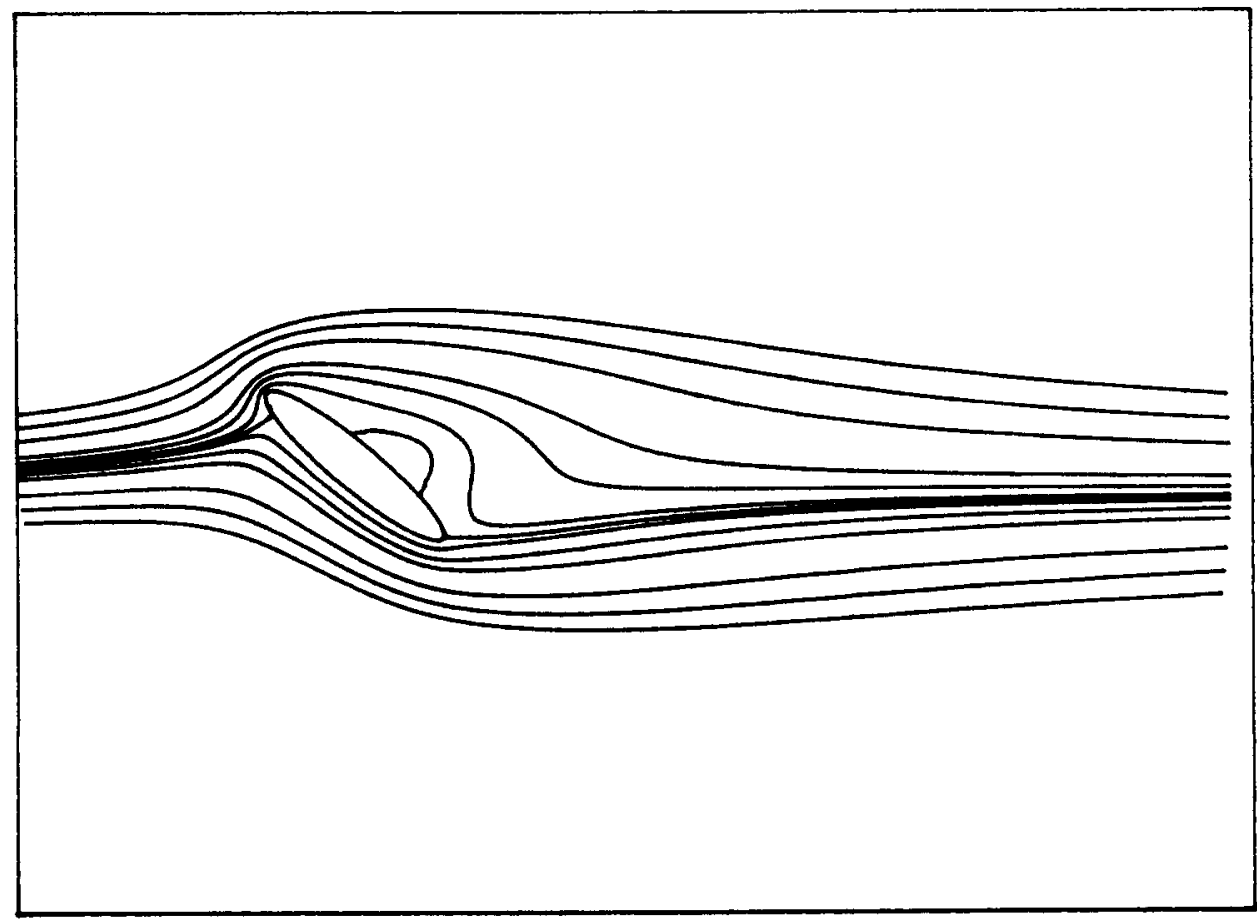

Fig. 6. Streamlines for $\operatorname{Re}^{\prime}=20, \alpha=40^{\circ}$ and $r=0.2$ for flow past an inclined elliptic cylinder. 


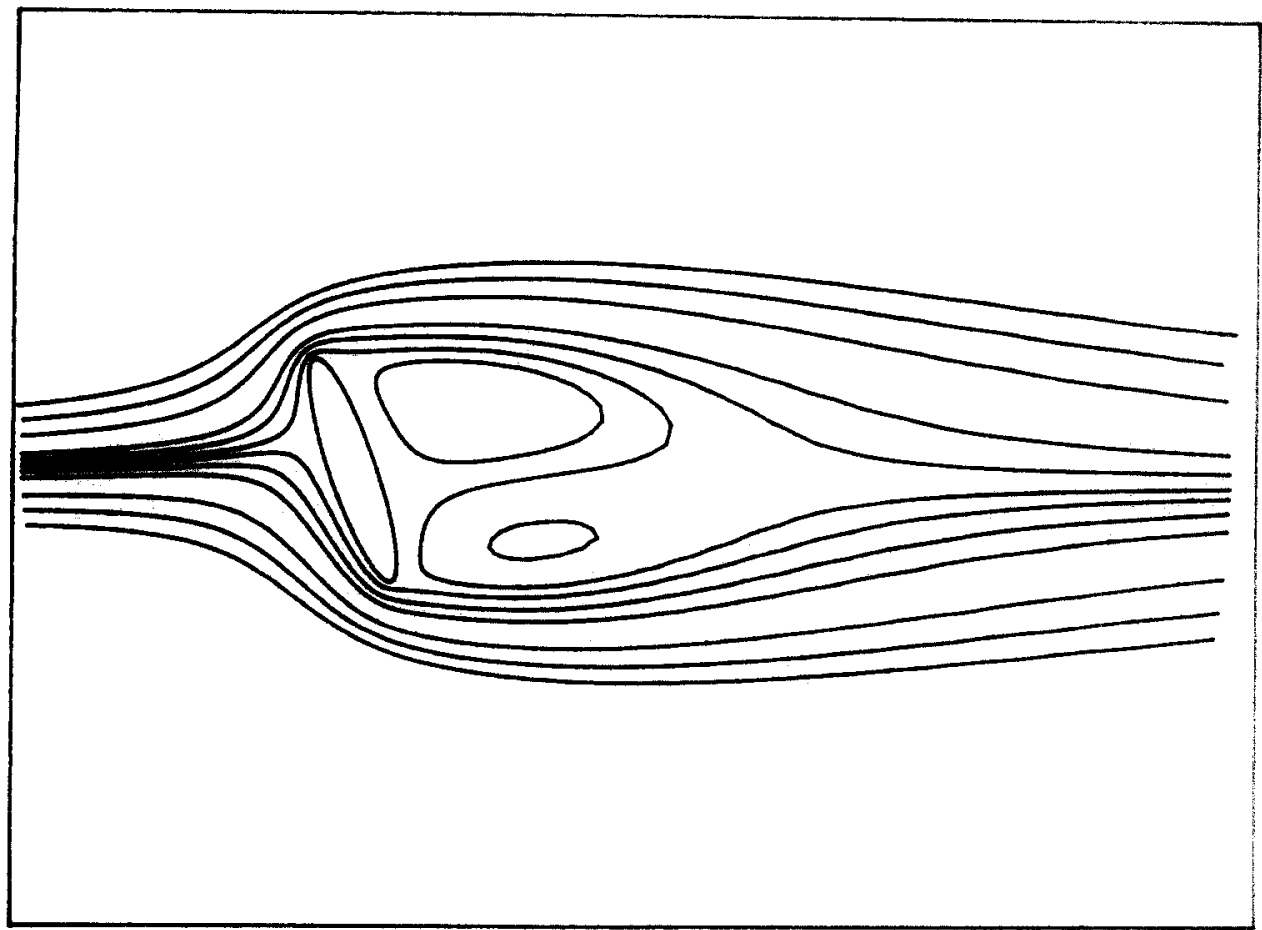

Fig. 7. Streamlines for $\operatorname{Re}^{\prime}=20, \alpha=70^{\circ}$ and $r=0.2$ for flow past an inclined elliptic cylinder

from the cylinder. Thus the asymptotic decay of the new dependent variable $\Phi$ is given by approximation (28), in which $P(\theta)$ is not a rapidly varying function and hence $\Phi$ has an expecially suitable form for approximation by finite-difference methods. Near the cylinder itself the function $F(\xi, \theta)$, given by equation (15), is not large and the behaviour of $\Phi(\xi, \theta)$ is similar to that of $\zeta(\xi, \theta)$. Several problems have been considered using the model, including two cases in which the flow is asymmetrical about the direction of undisturbed motion. In such cases it is important to enforce

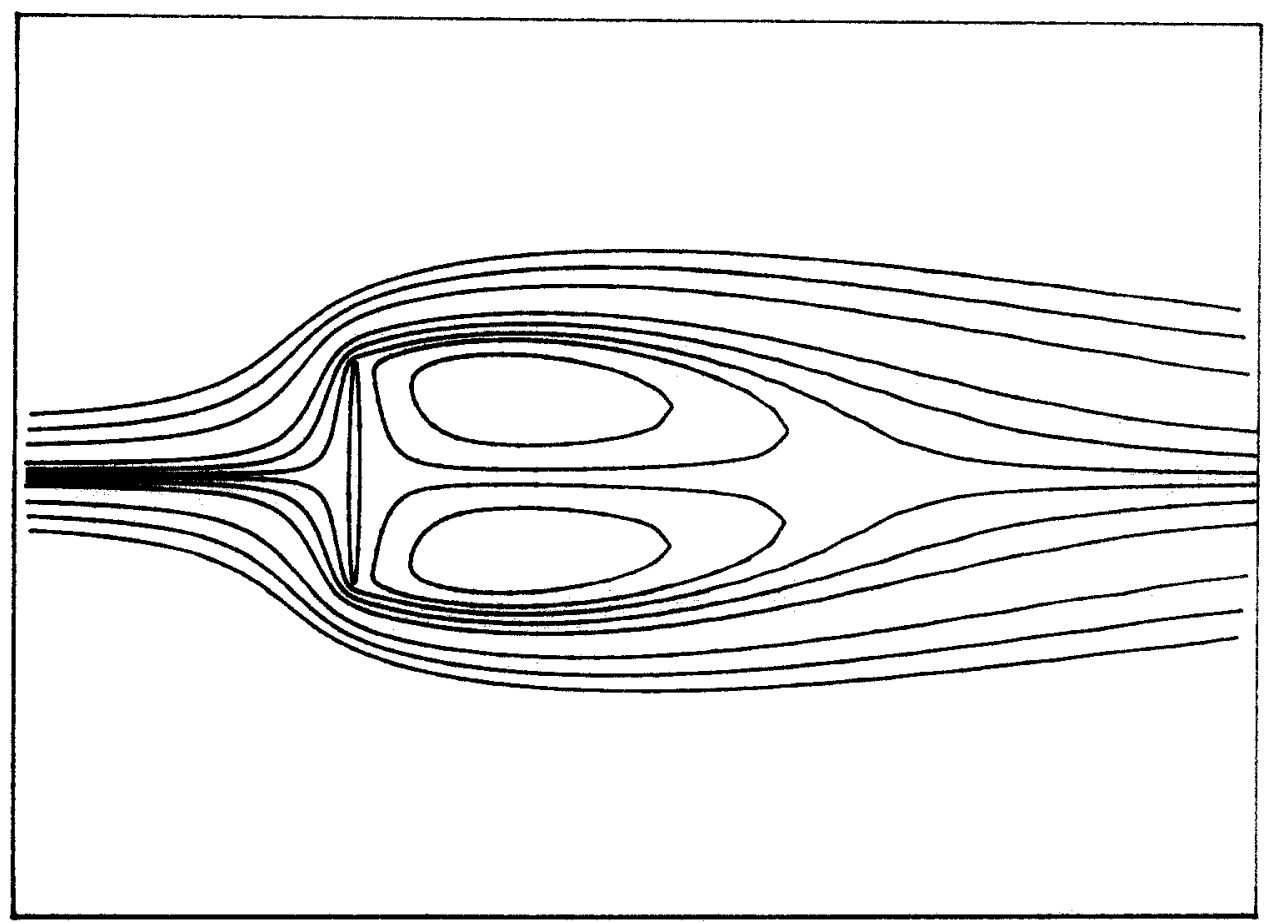

Fig. 8. Streamlines for $\operatorname{Re}^{\prime}=20, \alpha=90^{\circ}$ and $r=0.05$ for flow past a thin elliptic cylinder. 
the correct decay of vorticity at large distances, as pointed out in Refs $[2,12]$. Good comparison with the results given in these papers is found, particularly in the case of flow past the elliptic cylinder. Numerical solutions for higher $\mathrm{Re}^{\prime}$ values are possible; however, we did not attempt this, since our main object is simply to test the efficiency of the model.

Acknowledgement-Financial support for this research was provided by the Natural Sciences and Engineering Research Council of Canada.

\section{REFERENCES}

1. S. C. R. Dennis, The computation of two-dimensional asymmetrical flows past cylinders. Comput. Fluid Dynam. SIAM-AMS Proc. 11, 159 (1978).

2. H. M. Badr, S. C. R. Dennis and P. J. S. Young, Steady and unsteady flow past a rotating circular cylinder at low Reynolds numbers. Computers Fluids 17, 579 (1989).

3. I. Imai, On the asymptotic behaviour of viscous fluid flow at a great distance from a cylindrical body, with special reference to Filon's paradox. Proc. R. Soc. Lond. A208, 487 (1951).

4. B. Fornberg, Steady viscous flow past a circular cylinder up to Reynolds number 500. J. Comput. Phys. 61, 297 (1985).

5. M. Abramowitz and I. A. Stegun (Eds), Handbook of Mathematical Functions: Applied Mathematics Series-55, NBS, Washington, DC (1964).

6. S. C. R. Dennis and G.-Z. Chang, Numerical solutions for steady flow past a circular cylinder at Reynolds numbers up to 100. J. Fluid Mech. 42, 471 (1970).

7. B. Fornberg, A numerical study of steady viscous flow past a circular cylinder. J. Fluid Mech. 98,819 (1980).

8. T. P. Loc. Etude numerique de l'ecoulement d'un fluide visqueux incompressible autour d'un cylindre fixe ou en rotation. Effetmagnus. J. Mec. 14, 109 (1975).

9. D. B. Ingham, Steady flow past a rotating cylinder. Computers Fluids 11, 351 (1983).

10. D. B. Ingham and T. Tang, A numerical investigation into the steady flow past a rotating circular cylinder at low and intermediate Reynolds numbers. J. Comput. Phys. 87, 91 (1990).

11. T. Tang and D. B. Ingham, On steady flow past a rotating circular cylinder at Reynolds numbers 60 and 100. Computers Fluids 19, 217 (1991).

12. S. C. R. Dennis and P. J. S. Young, Steady flow past an elliptic cylinder inclined to the stream. J. Fluid Mech. (submitted).

13. S. C. R. Dennis and J. Dunwoody, The steady flow of a viscous fluid past a flat plate. J. Fluid Mech. 24, 577 (1966).

14. J. D. Hudson and S. C. R. Dennis, The flow of a viscous incompressible fluid past a normal flat plate at low and intermediate Reynolds numbers: the wake. J. Fluid Mech. 160, 369 (1985).

15. N. W. McLachlan, Theory and Applications of Mathieu Functions. Clarendon Press, Oxford (1947). 